Research Article

\title{
Application of Digital Image Correlation Technique for the Damage Characteristic of Rock-like Specimens under Uniaxial Compression
}

\author{
Jing Chai, ${ }^{1,2}$ Yongliang Liu $\mathbb{D}^{1},{ }^{1}$ YiBo OuYang, ${ }^{1}$ Dingding Zhang, ${ }^{1,2}$ and Wengang Du ${ }^{1}$ \\ ${ }^{1}$ School of Energy, Xi'an University of Science and Technology, Xi'an, Shaanxi 710054, China \\ ${ }^{2}$ Western Key Laboratory of Mine Mining and Disaster Prevention, Ministry of Education, \\ Xi'an University of Science and Technology, Xi'an, Shaanxi 710054, China
}

Correspondence should be addressed to Yongliang Liu; ylliu.xust@gmail.com

Received 1 August 2020; Revised 29 October 2020; Accepted 2 November 2020; Published 7 December 2020

Academic Editor: Zhi Cheng Tang

Copyright (c) 2020 Jing Chai et al. This is an open access article distributed under the Creative Commons Attribution License, which permits unrestricted use, distribution, and reproduction in any medium, provided the original work is properly cited.

The damage and degradation are the main influence factors of the instability of rock mass engineering. In this paper, the damage and deformation characteristics of the rock-like samples are investigated under the uniaxial compression test, and the advanced digital image correlation (DIC) device is devoted to full-field deformation data acquisition on the sample surface. Based on the full-field deformation data, a new damage variable is proposed by the principal strain standard deviation to characterize the uniaxial compression damage process of the rock-like samples. The results show that the newly presented damage variable can be utilized for the quantitative characterization of the sample damage. According to the characteristics of the damage variable, the damage evolution process of the rock-like specimens under uniaxial compression can be divided into four stages: initial damage closure stage, linear elastic damage stage, elastic-plastic damage stage, and plastic damage stage. From the stress-strain curve, the cut-off point from elastic to plastic deformation of the rock-like specimen is also the turning point from micro to macro damage; after the point, the apparent initial damage starts to occur on the sample surface; furthermore, the damage of the specimen is accelerated in the plastic damage stage. When the overall damage variable reaches 0.5 or the damage variable of strain localization zone reaches 0.8 , the macro crack forms, and the bearing capacity of the rock-like specimen decreases rapidly. The findings are of great significance to the prediction of the damage process of rock mass engineering by digital image correlation.

\section{Introduction}

With economic development in China, the construction of geotechnical engineering, such as dams, bridges, tunnels, and mining engineering, is in the ascendant. The damage and degradation of rock mass engineering are serious under complex occurrence conditions and stress environments. To assess the stability of rock engineering in real time, methods such as monitoring and identifying the damage, maintenance, and reinforcement of the critical region are utilized increasingly. They are critical for ensuring the safe and stable operation of rock engineering.

To reveal the failure mechanism of rock mass, the damage and degradation process of rock-like materials has been studied recently by many scholars with the theory of fracture mechanical behaviors, such as crack propagation, damage evolution process, and degradation and stability evaluation. A 0-1 variable scalar was initially established by Walsh to describe the damage evolution process of rock [1]. The concept of damage variable $D$ was proposed by Krajcinovic for the first time [2]; then, a generality damage model was presented by Horii based on the previous research results, which made an important contribution to the quantitative description of damage evolution of rock and other solid materials [3].

In the aspect of rock damage constitutive models, considering the joint geometry and mechanical properties of rock mass $[4,5]$, the deformation characteristics of jointed rock mass with different parameters under uniaxial, triaxial, and cyclic loads were studied, and the damage constitutive 
model of jointed rock mass under different loads was established [6-10]. Considering the influence of the occurrence environment on the deformation and damage process of rock $[11,12]$, the damage constitutive models of rock under the conditions of the freeze-thaw cycle, high temperature, and moisture were studied [13-17]. These studies have laid a solid foundation for mastering the law of damage and failure of rock.

With the popular application of acoustic emission, $\mathrm{X}$-ray, computed tomography $(\mathrm{CT})$, scanning electron microscopy (SEM), and so forth in rock mechanics, the researchers gradually integrate them with the damage theory and try to describe the damage process of rock mass from the micro and macro scale.

Under the loading, the internal defects of rock develop and form damage. In the process, the acoustic emission phenomenon is apparent. AE is very popularly utilized to detect the macro damage of rock. The damage state of rock under different loading conditions can be reflected by the spatial evolution characteristics of $\mathrm{AE}$ events $[18,20]$. To investigate the damage evolution process of rock, scholars combined the acoustic emission energy [20,21], events counts $[20,22]$, ringing counts $[22,23]$, and other characteristic parameters with the damage theory, establishing new damage variables characterizing the damage evolution law of rock.

The CT and SEM are widely used to identify the mesoscopic and microscopic damage characteristics and propagation process of rock cracks [23, 24]. Based on the principle of CT, a method of establishing the damage variable was put forward by CT number [25]. Compared with Bellonoi's and Lemaitre's formula, it can be applied to a wider range and reveal the damage mechanism of rock and concrete well. The damage variable based on the damaged area, such as crack and pore, is defined with the CT image processing technology [26], for instance, gray-level analysis and image segmentation technology, providing a feasible method for quantitative analysis of rock damage evolution. To investigate the damage process of rock, the volumetric porosity [27, 28] and accumulated plastic strain [29] are utilized to establish the damage variable combining the SEM. The mechanical behavior relationship between the micro and macro damage mechanisms of rock materials was investigated by observing the whole deformation and failure process with SEM [30].

As an advanced optical testing method, DIC is also combined with damage theory to evaluate the deformation and failure of rock. The shear strain, maximum shear strain variation coefficient, the gray-scale variation, and so forth obtained by DIC were used to propose the damage variable. The maximum shear strain, extracted on the samples with DIC, was used to establish a damage variable, which was utilized to describe the deformation and damage process of marble and slate with holes [31]. The gray-scale variation in the deformation process of the rock sample was studied by $\mathrm{Ma}$, and the relationship between the gray-scale variation and the damage was established to describe the damage evolution process of samples [32]. The strain localization process of coal samples was investigated by Wang et al.; the maximum shear strain variation coefficient was presented by extracting the surface shear strain [33]. The changes of strain gradient responding to loading were observed in the flat dog-bone specimens tensile tests with DIC [34]. DIC technique can be utilized to describe the evolution of damage in rock materials.

Compared with CT, SEM, and AE, DIC has the typical advantages of large test range, lower cost, high precision, full-field observation, and so forth. As an advanced nondestructive monitoring method, DIC has been widely used in the field of rock mechanics. Up to now, there are few researches on the evolution of rock-like samples damage by DIC. In this paper, based on the advantage of full-field strain measurement, the damage variable was defined with the main strain standard deviation for investigating the damage evolution process of rock-like samples. The findings are of great significance to the prediction of damage deterioration of rock engineering.

\section{Experimental Setup and Specimen Preparation}

2.1. $3 D$-DIC Setup. Digital image correlation (DIC) is a rising optical measurement technology in recent years. Due to the unique advantages, such as noncontact measurement, full-field strain and displacement data extracting, and high precision, it has been widely used. In this paper, the ARAMIS-3D commercial full-field strain test system of the GOM group is used. It is mainly composed of an industrial camera, digital image correlation calculation software, and calibration system.

2.1.1. Principle of 3D-DIC. The 3D-DIC is developed from $2 \mathrm{D}$-DIC. It is a $3 \mathrm{D}$ deformation measurement method that integrates the computer binocular stereo vision and 2D-DIC related matching technology. Based on the principle of binocular stereo vision, the system needs to be calibrated to obtain the internal and external parameters of the camera before the test. Usually, the process is that the two cameras of a certain angle to each other take several photos of the calibration plate with different attitudes. As shown in Figure $1(\mathrm{a})$, take the point $P\left(X_{W}, Y_{W}, Z_{W}\right)$ on the sample surface in the world coordinate system $\mathrm{O}\left(X_{W}, Y_{W}, Z_{W}\right)$ as an example, respectively, and the point $P$ is imaged at points of $P_{1}\left(U_{1}, V_{1}\right)$ and $P_{2}\left(U_{2}, V_{2}\right)$ in the image plane of the left and right cameras, respectively. From the knowledge of stereo vision [35], when the internal and external parameters of left and right cameras are known, the image coordinate $P(U, V)$ is uniquely determined for any given $3 \mathrm{D}$ space point $P\left(X_{W}\right.$, $\left.Y_{W}, Z_{W}\right)$, and the 3D coordinate of the point is also to be reverse-calculated. As a result, the $3 \mathrm{D}$ coordinate of point $\mathrm{P}$ is determined in the world coordinate system $\left(X_{W}, Y_{W}, Z_{W}\right)$.

The coordinate in the left and right images of the same point on the sample will be identified by binocular stereo vision technology when the 3D-DIC begins measurement. At the same time, the $2 \mathrm{D}$ digital image correlation technology is utilized to match the deformed and undeformed images of the sample. Note that the deformed image will be 


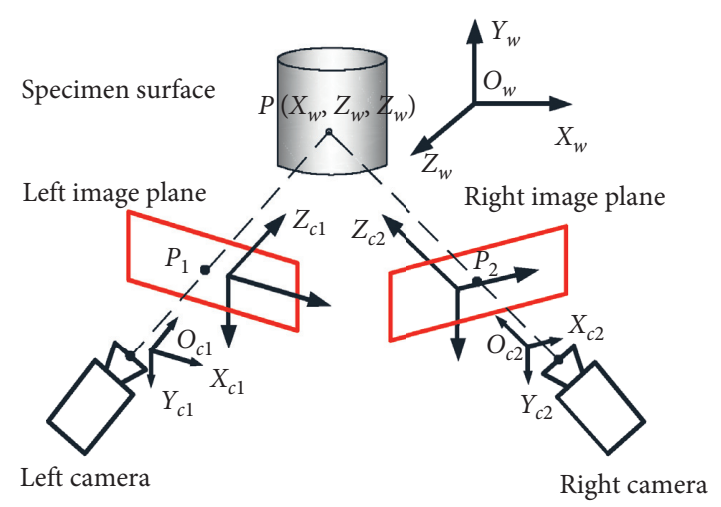

(a)

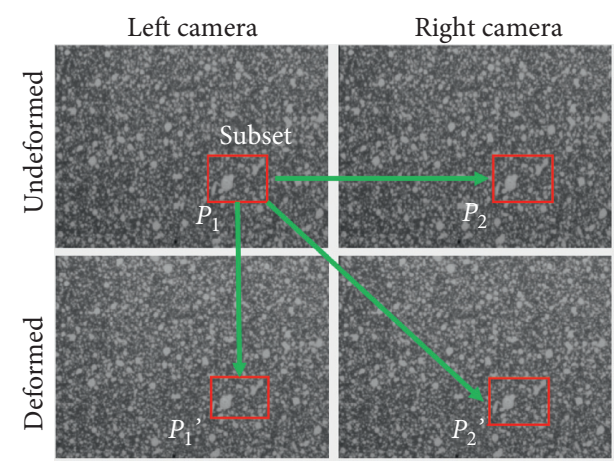

(b)

FiguRE 1: 3D-DIC measurement principle. (a) Binocular stereo vision principle. (b) Three-dimensional displacement calculation.

divided into some small grids named subsets in the matching process. It will be used to evaluate the correlation of deformed images. Generally, the correlation of subsets is calculated by the normalized covariance cross-correlation function shown in formula (1), where $f(x, y)$ and $G(x, y)$ represent the gray distribution of the subregion of the reference image and the target image, respectively. The points of the subregion center in the two images are completely matched when the correlation coefficient $C$ is up to 1 , and, also, the reference subregion is completely correlated with the target subregion:

$$
C=\frac{\sum_{i=-M}^{M} \sum_{j=-M}^{N}\left[f\left(x_{i}, y_{j}\right)\right]\left[g\left(x_{i}^{\prime}-y_{j}^{\prime}\right)-g_{m}\right]}{\sqrt{\sum_{i=-M}^{M} \sum_{j=-m}^{N}\left[f\left(x_{i}, y_{j}\right)-f_{m}\right]^{2}} \cdot \sqrt{\sum_{i=-M}^{M} \sum_{j=-m}^{N}\left[g\left(x_{i}^{\prime}, y_{j}^{\prime}\right)-g_{m}\right]^{2}}} .
$$

In 3D-DIC deformation measurement, the matching procedure of image before and after deformation in the left and right cameras is represented by Figure $1(\mathrm{~b})$. Point $P(X w$, $Y w, Z w)$ in space is imaged on points $P_{1}\left(u_{1}, v_{1}\right)$ and $P_{2}\left(u_{2}\right.$, $v_{2}$ ) on the left and right cameras, respectively, before deformation. After deformation, point $P$ is moved to $P^{\prime}(X w$, $Y w, Z w)$, and the points $P_{1}\left(u_{1}, v_{1}\right)$ and $P_{2}\left(u_{2}, v_{2}\right)$ on the image plane of left and right cameras are imaged, respectively. The correlation function of equation (1) is used to calculate the correlation of images. The image subregion of the right camera before deformation will be taken as the reference subregion. To confirm the point $P$ in the deformed image, the subregion with the highest correlation between the deformed image and the reference image will be matched. The displacement from $P^{\prime}$ to $P$ could be obtained by the three-dimensional coordinate. Repeat the above process for all pixels in the image, and then the three-dimensional displacement field of the sample surface can be obtained. After the displacement is smoothed, the corresponding strain field can be obtained by differential calculation.

2.1.2. 3D-DIC Image Acquisition Method. As shown in Figure 2, the ARAMIS 3D-DIC image acquisition system is mainly composed of two COMS industrial cameras, two fixed-focus lenses with a focal length of $50 \mathrm{~mm}$, two highbrightness blue-light sources without stroboscopic light, and a computer with data acquisition. The resolution of COMS is
$4096 \times 3000$. During the test, the sample should be filled with camera vision as much as possible.

DIC collects two photos per second during the uniaxial compression of rock-like samples. The subset is 19 pixels and the spacing is 16 pixels in the data processing stage. The actual length of each pixel is calculated to be $0.5 \mathrm{~mm} /$ pixel in the experiment. A ROI (region of interest) $40 \mathrm{~mm}$ wide $\times 80 \mathrm{~mm}$ high is set on the specimens to obtain the data strain and displacement.

\subsection{Sample Preparation and Test Procedure}

2.2.1. Sample Preparation. Based on the parameters of sandstone in the field, three kinds of rock-like samples were made with quartz sand, iron powder, barite powder, gypsum, and alcohol rosin solution. Quartz sand accounts for $30 \%$ of the total weight of rock-like materials. Gypsum content is the ratio of gypsum to total materials. $40 \mathrm{~g}$ alcohol is needed for every $1000 \mathrm{~g}$ of rock-like materials. The binder concentration is the ratio of rosin to rosin and alcohol solution weight, and the weight of barite powder and iron powder is $70 \%$ of the total similar material weight. In order to summarize the damage evolution law of the rock-like material under uniaxial compression, the three kinds of samples with different proportions were made. The mechanical parameters of rock-like sample and the field sandstone are shown in Table 1. The contents of iron powder, alcohol rosin 


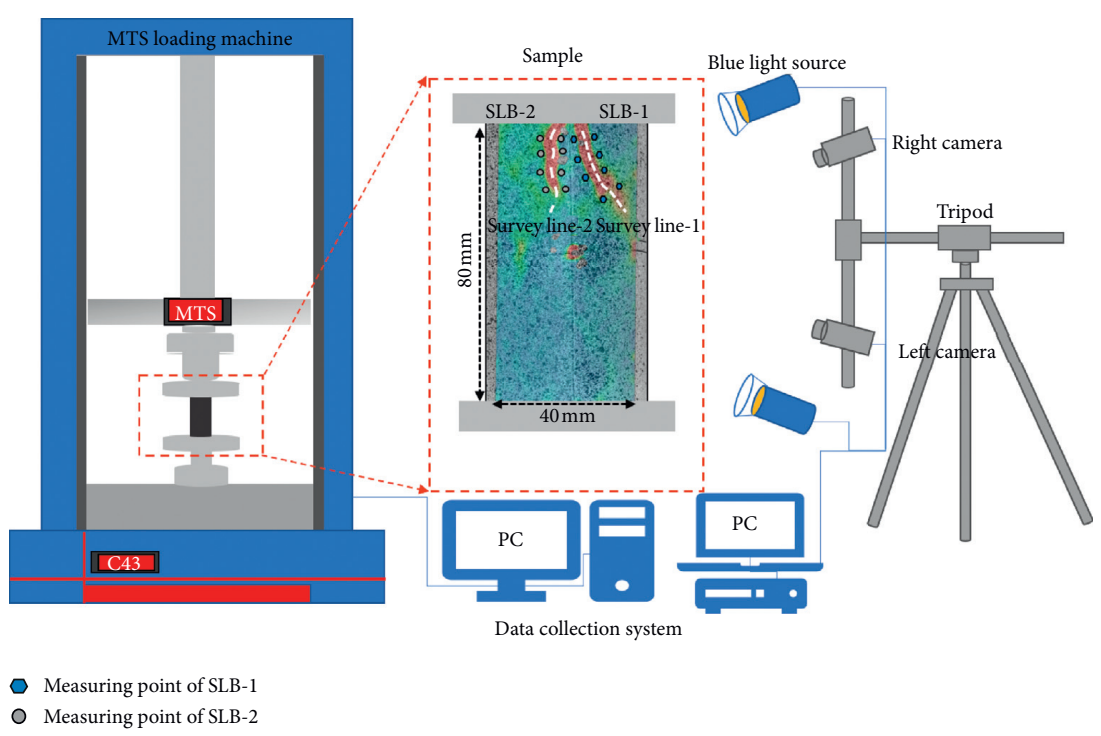

Figure 2: Test system diagram.

Table 1: Proportion and mechanical parameters of the sample.

\begin{tabular}{|c|c|c|c|c|c|c|c|c|c|c|}
\hline Number & A (\%) & B (\%) & $\mathrm{C}(\%)$ & $\mathrm{D}(\mathrm{MPa})$ & Density $\left(\mathrm{g} * \mathrm{~cm}^{3}\right)$ & UCS (MPa) & $\mathrm{E}(\mathrm{GPa})$ & $\sigma_{t}(\mathrm{MPa})$ & Poisson's ratio & Brittleness (\%) \\
\hline$S 1$ & 20 & 25 & 8 & 6.0 & 2.07 & 2.63 & 3.06 & 0.19 & 0.17 & 7.2 \\
\hline$S 2$ & 35 & 25 & 6 & 4.0 & 2.18 & 2.31 & 2.87 & 0.17 & 0.15 & 7.3 \\
\hline$S 3$ & 30 & 20 & 8 & 2.0 & 2.04 & 2.14 & 6.26 & 0.15 & 0.19 & 7.0 \\
\hline \multicolumn{5}{|c|}{ Field sandstone } & 2.32 & 27.5 & 35.31 & 1.93 & 0.21 & 7.0 \\
\hline
\end{tabular}

solution, gypsum, and the forming pressure of the material are represented by $A, B, C$, and $D$. A high-accuracy electronic scale of $0.01 \mathrm{~g}$ was utilized to weight the material, such as iron powder, barite powder, quartz sand, rosin, and alcohol. To ensure the homogeneity of the samples, the material will be fully stirred and then loaded into the mold and formed on the universal testing machine according to the set forming pressure. Finally, according to the standard for test methods of engineering rock mass (GB/T 50266-2013) [36], the cylindrical standard specimens with a height of $100 \mathrm{~mm}$ and a diameter of $50 \mathrm{~mm}$ are produced.

2.2.2. Test Procedure. The uniaxial compression experiment was conducted by the high-precision MTS electrohydraulic servo testing machine with a range of $50 \mathrm{kN}$. For the principle of DIC, the sample's surface needs to make some speckle of a diameter of about $1 \mathrm{~mm}$ with black and white paint before the test. After the speckle was made, placing the sample on the MTS, the angle of the sample is adjusted to present the speckled surface in the field of vision of two cameras. The loading mode of MTS is displacement control; the loading rate and the sampling frequency are set separately as $1 \mathrm{~mm} / \mathrm{min}$ and $2 \mathrm{~Hz}$, respectively. Meanwhile, to conveniently compare the data, the acquisition rate of the camera is also set to 2 pieces per second, and the devices start to measure at the same time.

\section{Failure Characteristics of Rock-like Samples}

3.1. Typical Stress-Strain Curve of Rock Sample. The stressstrain curves of $S 1, S 2$, and $S 3$ are shown in Figure 3.

As shown in Figure 3, the UCS (Uniaxial Compressive Strength) of samples $S 1, S 2$, and $S 3$ is around 2.5 MPa. The stress of the sample drops rapidly after reaching the peak value.

Brittleness is an important parameter for evaluating rock-like materials. At present, it is usually evaluated by the tension-compression ratio, and the smaller the ratio, the stronger the brittleness of the rock [37]. The tensioncompression ratio of natural rock is approximately $2.9 \%$ to 8.3\% [38]. According to the relevant parameters of $S 1-S 3$ in Table 1, the tension-compression ratio of rock-like sample is from $5.9 \%$ to $7.1 \%$, so the brittleness of the rock-like sample in this paper is similar to natural rock. The rock-like material selected in the experiment has good performance to simulate the rock.

3.2. Failure Characteristics of Rock Samples. In this section, taking $S 1$ as an example, the deformation and failure modes of rock-like samples are analyzed. In the test, the specimen $S 1$ first produced macro cracks from the right side of the specimen top. With the continuous increase of stress, the left side of the specimen also produced an $X$-shaped crack intersecting the right side, and the final specimen presented 


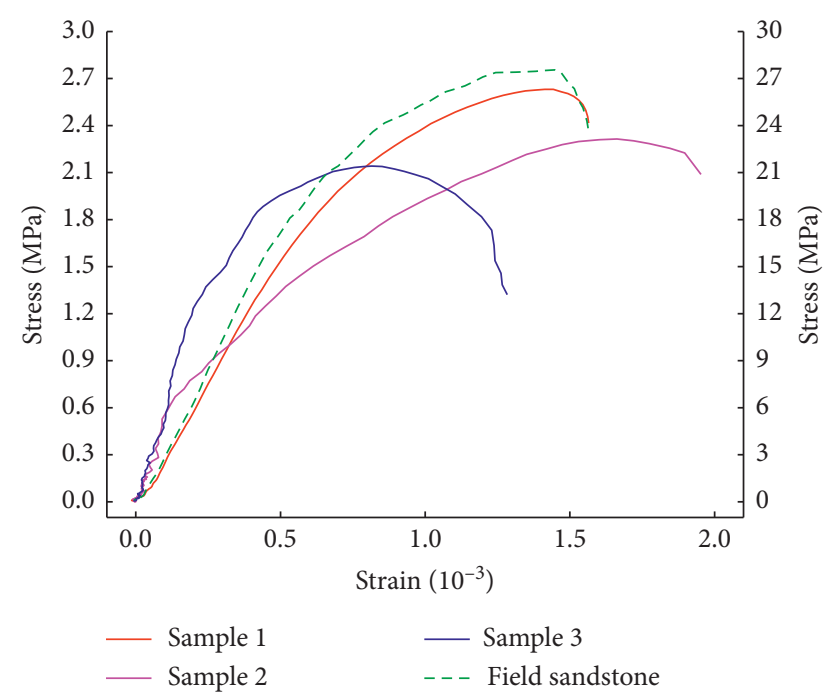

FIGURE 3: Stress-strain curve of uniaxial compression.

an $X$-shaped failure. The characteristics main strain and displacement were analyzed by selecting 10-key-point nephogram of ROI on the sample $S 1$. Figure 4 is the main strain evolution cloud diagram of ROI (region of interest) on the sample surface before the peak stress of $S 1, S 2$, and $S 3$.

To analyze the failure characteristics of the specimen, measure points located at both sides of the strain localization band (SLB) are evenly arranged, which are to extract the data of the main strain and displacement in the loading process in the axial ( $Y$ direction) and radial ( $X$ direction) directions of the sample.

From the results obtained in Table 2, before the peak stress up to $0.3 \sigma_{c}$, there is a small strain concentration point scattered sporadically on the surface of the sample $S 1$. When the peak stress is between $0.4 \sigma_{c}$ and $0.6 \sigma_{c}$, the points of strain concentration on the surface of the specimen increase and gradually develop into a short strain localization band; with the further increase of stress, the strain localization band continues to grow and connect, forming a relatively clear strain localization band.

When the stress reaches $0.6 \sigma_{c}$, two more obvious $X$ shaped strain localization bands are formed at the upper end of the sample. When the stress increases from $0.7 \sigma_{c}$ to $\sigma_{c}$, the $X$-type strain localization band in the upper part of the specimen continues to extend to the lower part of the specimen, the width increases gradually, and the localization bands in other positions are gradually connected into a network, staggered distribution. When the peak stress is reached, two obvious strain localization bands are formed on the surface of the upper end of the specimen, the position is consistent with the failure crack shape of the specimen, and the strain concentration degree of the localization band on the right side of the upper end is greater than that on the left side.

Four measuring points were arranged separately on both sides of $S 1$ localization bands 1 and 2, as shown in Figure 2. The displacement, extracted, respectively, in $X$ and $Y$ directions of the points, was utilized to describe the failure

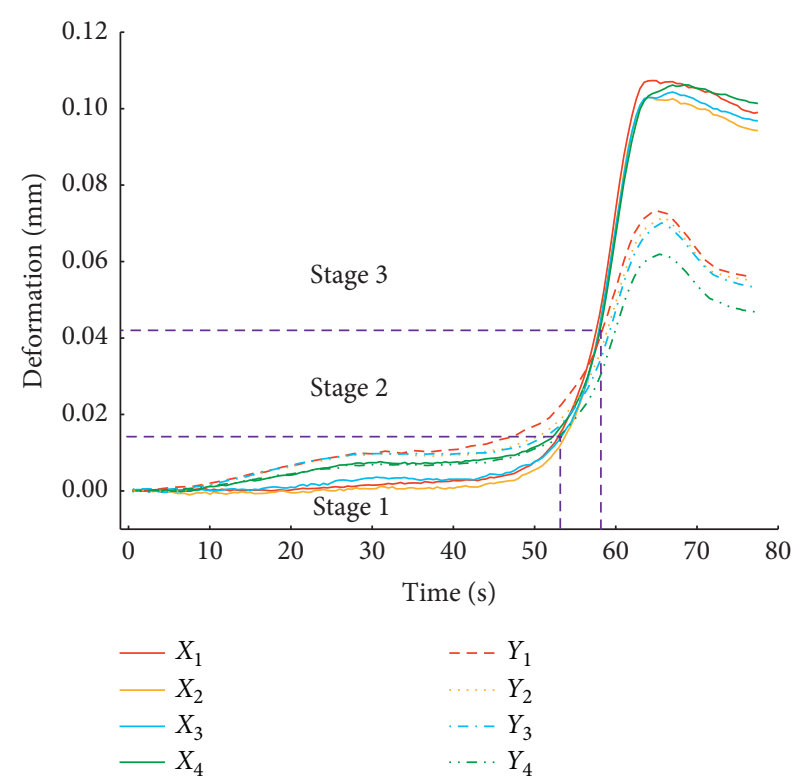

FIGURE 4: Displacement evolution of measuring point in SLB1.

mode of samples. The type of crack is determined by the relative value of the axial and radial displacement of the measuring points on both sides of the strain localization zone in this paper. When the radial deformation of the sample is greater than the axial deformation, it is a tensile crack; and when it is not greater than the axial deformation, it is a shear crack. The results are shown in Figures 4 and 5. Note that the downward compression displacement of the sample is positive.

As shown in Figure 4, the deformation of $S 1$ localized band 1 can be divided into three stages. In the first stage, the axial ( $Y$ direction) displacement of localized band 1 is larger than that of radial ( $X$ direction) displacement before the specimen is loaded for $53 \mathrm{~s}$, and the strain localization is mainly shear deformation. In the second stage, the specimen is loaded with $53 \mathrm{~s}-56 \mathrm{~s}$, and the axial and radial displacements of the specimens increase rapidly until the radial and axial displacements are the same. In the third stage, after $59 \mathrm{~s}$ of loading, the radial displacement of the specimen is obviously larger than that of the axial displacement, and the macroscopic crack occurs. The cracks formed in the strain localization band 1 of the specimen are mainly subjected to shear crack.

As shown in Figure 5, the displacement evolution of the measuring points on both sides of the strain localization zone 2 can also be divided into three stages. Stage 1 is $53 \mathrm{~s}$ before loading, and the axial displacement of localization zone 2 is larger than that of radial displacement, which is shear deformation. In the second stage, the specimen was loaded with $53 \mathrm{~s}-63 \mathrm{~s}$, and the radial displacement of the localized zone 2 was larger than that in the axial direction, which was tensile deformation. In stage 3 , after the specimen was loaded for $63 \mathrm{~s}$, the tensile displacement of localization band 2 further increased, which led to the failure of the sample. Therefore, the crack in the localized band 2 is the first shear, and then tension formed. 


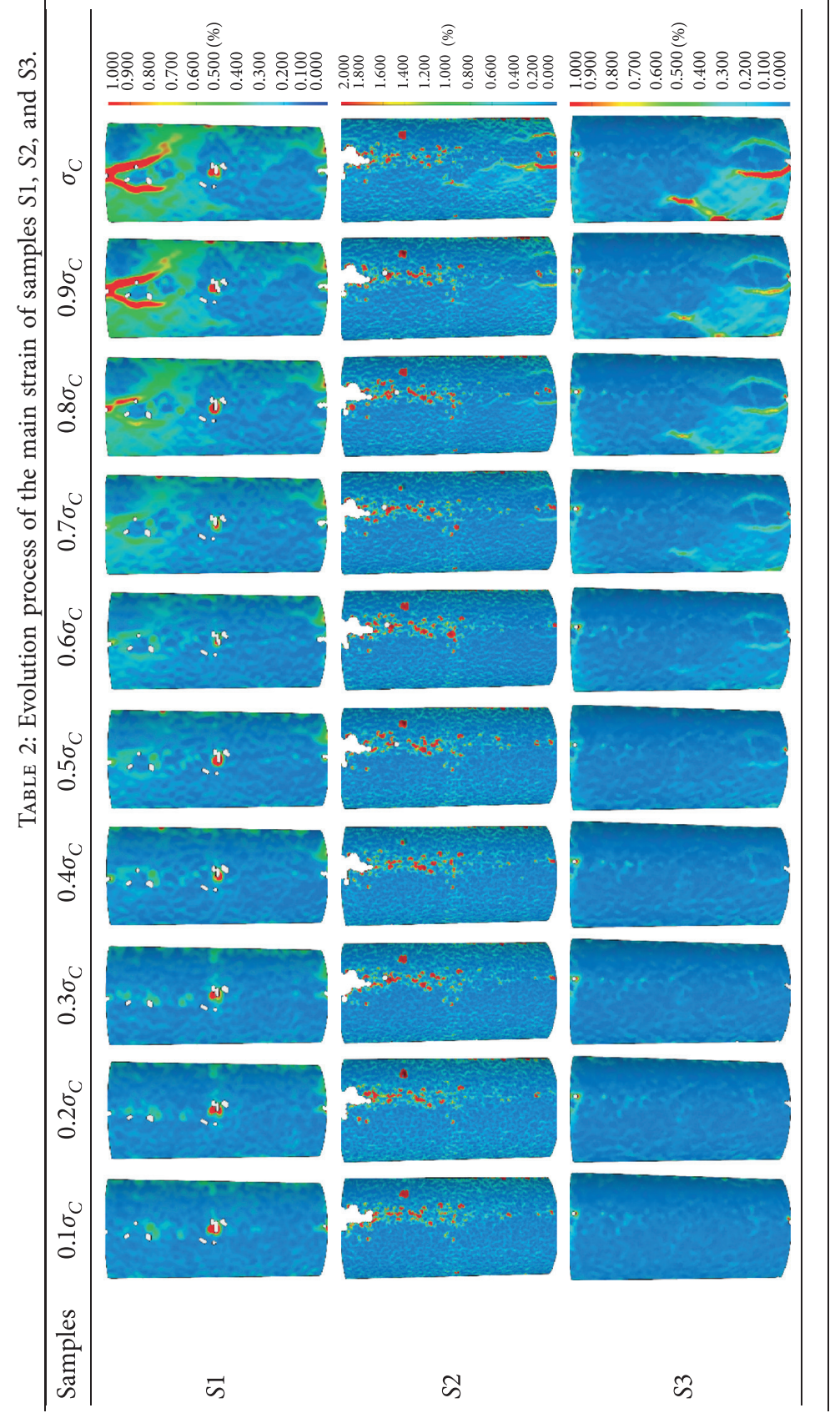




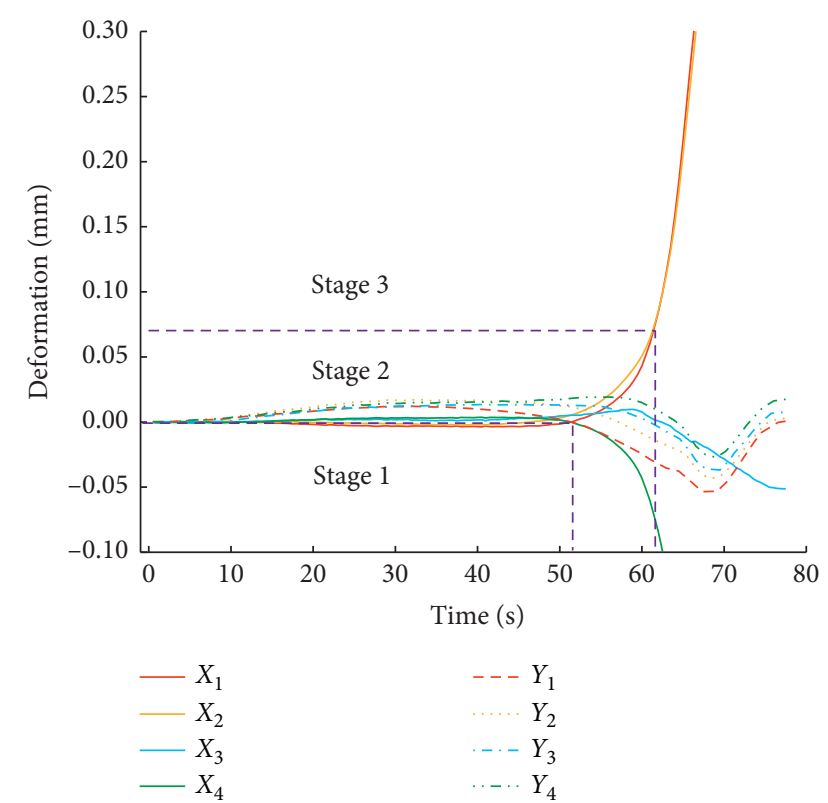

Figure 5: Displacement evolution of measuring point in SLB2.

\section{Damage Assessment of Rock-like Samples Based on 3D-DIC}

4.1. Establishment of Damage Variables. In the field of rock mechanics, defining new damage variables combining the parameters obtained from DIC with the damage theory is a key factor to describe the damage mechanism of materials.

According to the damage theory, the original damage variable is represented by the reduction of the effective bearing area. So the initial damage variable can be defined as in formula (2).

$A_{d}$ is the defect area on the effective bearing area, and $A$ is the effective bearing area.

$$
D=\frac{A_{d}}{A}
$$

In this paper, the standard deviation of the main strain is introduced into the formula of the damage variable. $S$ is the standard deviation of the main strain when the damaged area reaches $A_{d}$, and $S_{\max }$ is the main strain when the damaged area reaches $A_{d}$. Then formula (2) can be rewritten as

$$
D=\frac{S_{i}}{S_{\max }} .
$$

$S_{i}$ is the standard deviation of the main strain on each measuring line in formula (3), as shown in the following formula:

$$
S_{i}=\sqrt{\frac{1}{n-1} \sum_{1}^{n}\left(X_{i}-\bar{X}\right)^{2} .}
$$

In formula (4), $n$ is the number of statistical points, $X_{i}$ is the principal strain of $(i)$ statistical points at any time, and $\bar{X}$ is the average value of the principal strain on the measuring line.

The load was stopped when the load decreased to $20 \%$ of the peak load during the experiment. As a result, the specimen was not completely damaged and the damage variable did not reach 1 .

4.2. Data Extraction Method. A clear evolution nephogram of the displacement field and strain field in the process of specimen loading can be obtained with DIC technology. Strain and displacement data in all directions, by setting an ROI area on the measuring area and arranging lines and measuring points in the ROI area, can be extracted at any time in the process of sample loading. The maximum, minimum, and average strain data of the ROI region can also be obtained utilizing the statistical function of the software of DIC.

The ROI region is determined according to the research objective. Arranging the measuring line and point according to the location of SLB in the ROI area on the sample, the strain data can be extracted.

At the moment before the macro and micro cracks appear in sample $S 1$, there are many clear SLBs on the surface. The two SLBs of the sample are named SLB1 and SLB2. Measuring lines 1 and 2 are arranged along the propagation direction of SLB1 and SLB2, respectively. Strain data in the measuring lines are extracted to analyze the evolutionary relationship between the sample $S 1$ stressstrain curve and SLB1 and SLB2 damage variables. The measuring line and point arrangement in the ROI area of sample $S 1$ are shown in Figure 2.

\subsection{Damage Characteristics of Rock-like Sample}

4.3.1. Whole Damage Analysis of Sample. In this section, the damage evolution processes of $S 1, S 2$, and $S 3$ specimens were compared and analyzed with their stress-strain curve. As shown in Figure 6, taking $S 1$ as an example, five key points $O$, $A, B, C$, and $D$ were taken from the stress-strain curve of sample $S 1$ for analysis. The damage evolution process of $S 1$ specimen under uniaxial compression before peak stress can be divided into four stages.

(1) The samples in the stage of OA have uniform deformation. The microfissure and micropore in the samples are gradually compacted under the loading, and the damage variable tends to 0 , which is the initial damage closure stage.

(2) The microfissure and micropores had been compacted in the $\mathrm{AB}$ stage of sample deformation, and the deformation of the samples has entered the stage of linear elasticity. At this time, the internal micro damage of the specimen developed gradually, which was the stage of linear elastic damage. In this stage, the damage variable of apparent was still small, and the damage degree of the specimen was low. The deformation of the specimen changed from linear elastic to nonlinear elastic when the specimen was 


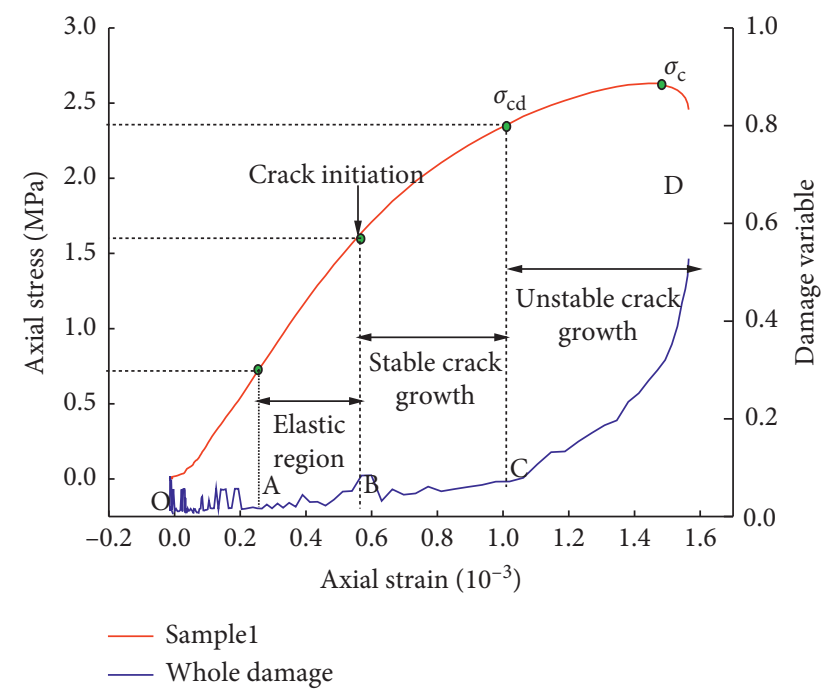

FIgURE 6: The relationship between whole damage variable and loading curve of sample $S 1$.

loaded to point B. The damage of the specimen increased and the damage variable began to evolve.

(3) The BC segment of sample deformation was the elastic-plastic stage. The segment microfissure developed stably, the damage of the specimen gradually evolved from the inside and outside to the surface, and the apparent damage variable gradually increased, which also is the elastic-plastic damage stage.

(4) Point $C$ is the critical point of the deformation of the sample from elastic-plastic to plastic. The fissure initiated and developed rapidly in the CD segment. Meanwhile, the damage variable increased quickly. It was the plastic damage stage, in which the specimen gradually develops from micro to macro failure. When the loading is up to the peak stress (point $D$ ), the macro cracks appeared, the whole damage variable of the specimen reached 0.528 , and the bearing capacity decreased rapidly.

In the OA segment, the damage variable of the $S 1$ sample fluctuated a little, which was caused by the error caused by the DIC system noise. It did not affect the whole damage evolution trend of the sample $S 1$.

As shown in Figure 7, when the loading of the specimen $S 1$ reached the peak stress, the whole damage value was 0.528 , which was a little small. The main reason is that the deformation of the specimen is nonuniform, leading to appearance of strain concentration area. The strain is more than the other regions in the area. So, the maximum principal strain is located in the strain localization zone, and $S_{\max }$ is larger, resulting in the whole damage of the specimen becoming smaller.

The failure of brittle materials, such as rock, is usually due to the inhomogeneous deformation of the specimen caused by the internal defects of the rock mass. The strain localization zone usually appears in the location of inhomogeneous deformation and where deformation is large and

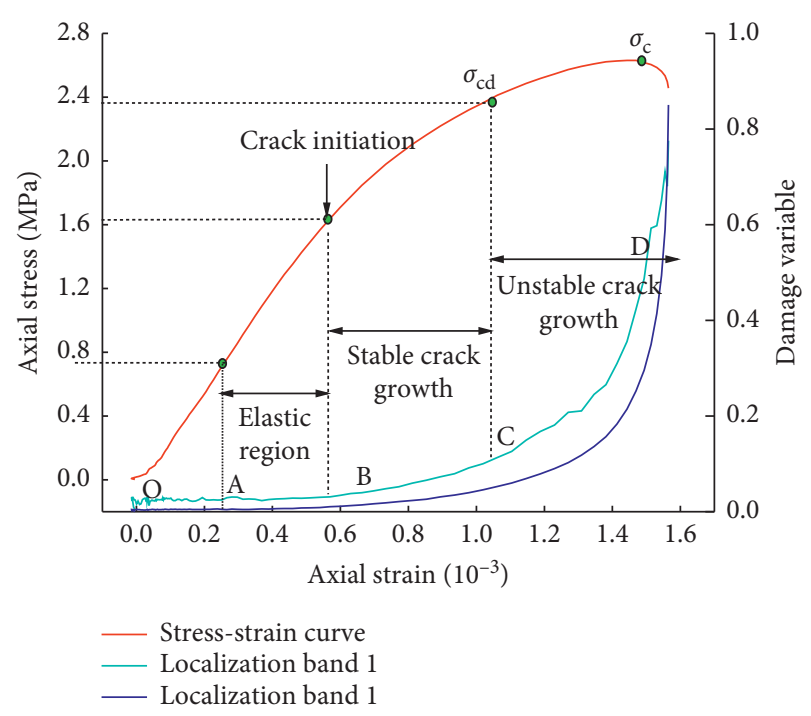

FIgURE 7: The relationship between damage variable and loading curve of SLB1.

easy to produce macro cracks, which leads to the whole failure of the specimen.

In this section, the damage evolution law of the whole ROI area was analyzed with DIC, which was the whole trend of the sample damage. However, the deformation of the specimen is nonhomogenous, as mentioned above, where the deformation of SLB is larger than the other zone. Therefore, to more carefully investigate the damage process of the specimen, the damage evolution process of the SLB was analyzed by the main strain distribution nephogram of the specimen $S 1$ before the appearance of macroscopic crack.

4.3.2. Damage Evolution Analysis of SLB. As shown in Figure 2, measuring lines 1 and 2 are arranged, respectively, for obtaining the principal strain and maximum standard deviation of the principal strain at the SLBs of sample S1. The data will be used for calculating the damage variable with equation (3).

It can be seen from Figure 7 that the damage evolution process of SLB1 and SLB2 can also be divided into four stages: the OA segment is the initial damage closure stage and is also the micro cracks compaction stage of the specimen. Meanwhile, the apparent damage is small, about 0.005 .

The $\mathrm{AB}$ segment is in the stage of linear elastic damage. The specimen is elastically deformed and the micro cracks propagate initially. At the same time, the sample's damage develops from the inside to the surface and evolves on the surface. It increases approximately linearly for the damage variables of SLB1 and SLB2, up to 1.3 and 3.6 times, respectively. The BC segment is an elastic-plastic damage stage. In this stage, the micro cracks develop steadily, and the damage variable of SLB1 and SLB2 increases quickly, up to 1.8 and 2.45 times. The damage of SLB1 and SLB2 is larger than the whole sample. The CD segment is the plastic damage stage. The microfractures rapidly unstably develop and connect to each other. The damage variables of SLB1 and 


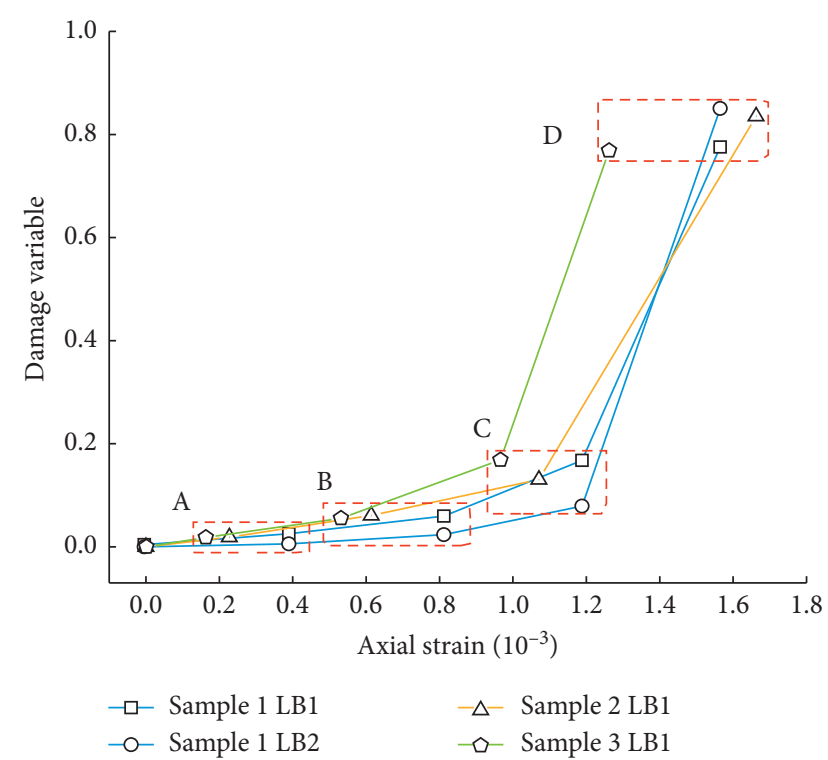

FIgURE 8: Damage evolution characteristic curve of SLB of specimens $S 1, S 2$, and $S 3$.

SLB2 are exponentially increased by 9.7 and 3.6 times larger than point $C$, respectively. It reaches the maximum value at point $D$ before the specimen failure.

The principal strains of $S 2$ and $S 3$ for analyzing damage were also obtained with DIC. The damage variable of the characteristic point of the stress-strain curve is obtained from the whole and SLB of specimens $S 1, S 2$, and $S 3$. It is shown in Figures 8 and 9, and more detailed data are shown in Table 3. It can be seen from Figure 8 that, before point $C$, the damage of SLB of the rock-like specimen is about 0.2 , and after reaching the point $D$, it is about 0.8 . The damage of the specimen in the on-line elastic stage is small, and the failure of the specimen in the yield stage is accelerated, and the larger macro failure is formed when the peak stress is reached.

It can be seen from Figure 9 that the whole damage of the sample highly increases in the $\mathrm{CD}$ segment. The damage variable of points $C$ to $D$ increases from 0.2 to 0.5 and the macro cracks and failure appeared in the stage. It could be illustrated that the rock-like specimen is stable before reaching the yield stress, and the damage in the yield phase is the main reason for the failure of the specimen. It is more than $60 \%$ of the total damage of the sample. The damage in the $\mathrm{AB}$ and $\mathrm{BC}$ segments is about $40 \%$ of the total of the sample, developed slowly, which has little impact on the stability of the sample.

Based on DIC obtained strain data, the damage variable defined with the standard deviation of principal strain can describe effectively the damage process of the specimen by analyzing the damage evolution process of $S 1, S 2$, and $S 3$. The damage evolution process of the samples can be divided into four phases at prepeak stress: initial damage closure stage, linear elastic damage stage, elastic-plastic damage stage, and plastic damage stage, corresponding to the compression stage, linear elastic stage, elastic-plastic stage, and yield stage of the stress-strain curve. The damage

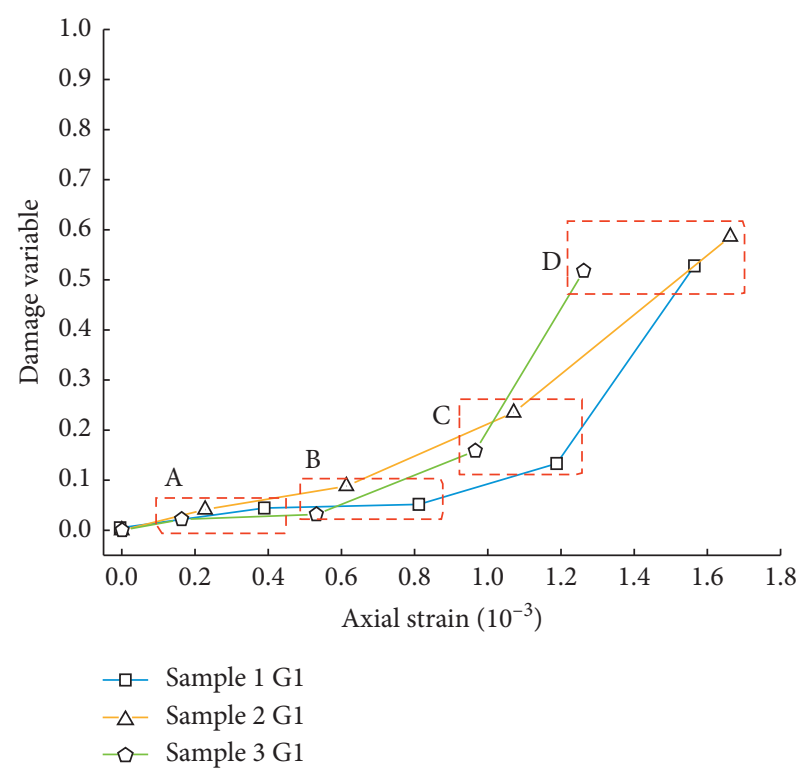

FIgURE 9: Damage evolution characteristic curve of whole specimens $S 1, S 2$, and $S 3$.

TABLE 3: Evolution process of damage variable of samples $S 1-S 3$.

\begin{tabular}{ccccccccc}
\hline \multirow{2}{*}{ Sample } & \multirow{2}{*}{ Damage } & \multirow{2}{*}{$A$} & \multirow{2}{*}{$B$} & \multirow{2}{*}{$C$} & \multirow{4}{c}{ Damage variation } \\
& & & & & & $\mathrm{AB}$ & $\mathrm{BC}$ & $\mathrm{CD}$ \\
\hline \multirow{4}{*}{$S 1$} & Global & 0.044 & 0.052 & 0.133 & 0.528 & 0.182 & 1.558 & 2.970 \\
& 1 & 0.026 & 0.060 & 0.168 & 0.776 & 1.308 & 1.800 & 3.619 \\
& 2 & 0.005 & 0.023 & 0.079 & 0.851 & 3.600 & 2.435 & 9.772 \\
& $\varepsilon / 10^{-3}$ & 0.390 & 0.812 & 1.188 & 1.565 & - & - & - \\
\hline \multirow{4}{*}{$S 2$} & Global & 0.041 & 0.088 & 0.235 & 0.586 & 1.146 & 1.670 & 1.494 \\
& 1 & 0.019 & 0.061 & 0.130 & 0.835 & 2.250 & 1.145 & 5.410 \\
& $\varepsilon / 10^{-3}$ & 0.228 & 0.614 & 1.071 & 1.663 & - & - & - \\
\hline \multirow{3}{*}{$S 3$} & Global & 0.022 & 0.032 & 0.159 & 0.518 & 0.455 & 3.969 & 2.258 \\
& 1 & 0.018 & 0.055 & 0.169 & 0.769 & 2.072 & 2.043 & 3.560 \\
& $\varepsilon / 10^{-3}$ & 0.164 & 0.532 & 0.966 & 1.262 & -- & -- & - \\
\hline
\end{tabular}

propagation of the specimen is a process from inside to outside. The characteristic point $B$, from linear elastic to nonlinear elastic deformation of the specimen, is the key point from internal to external damage evolution. Then, the damage of the specimen gradually evolves from internal to apparent scale.

\section{Conclusions}

(1) Using DIC technology to extract displacement data, through analyzing the displacement evolution of both sides of S1 SLB, it is shown that the position of S1 SLB1 is a shear crack, and the position of SLB2 is tensile crack. The failure mechanism of the sample is shear failure first and then tensile failure. The deformation failure mode of the specimen can be explained quantitatively with DIC.

(2) The whole field strain is obtained by DIC measurement technology, and the damage variable based on the standard deviation of principal strain is 
established. The damage evolution process of rocklike specimens can be divided into four stages: initial damage closure stage, linear elastic damage stage, elastic-plastic damage stage, and plastic damage stage. It is verified that the damage variable proposed can well describe the deformation and damage process of the specimen.

(3) The deformation of rock-like samples is inhomogeneous. The damage of the SLB zone is greater than that of the whole. The damage of rock-like samples is mainly concentrated in the yield stage. When the overall damage of rock-like samples reaches about 0.5 or the damage of the SLB zone reaches about 0.8 , the failure of samples will occur soon. The description of the rock damage process with SLB damage is more effective.

\section{Data Availability}

The data used to support the findings of this study are included within the article.

\section{Conflicts of Interest}

The authors declare that they have no conflicts of interest regarding the publication of this paper.

\section{Acknowledgments}

This study was financially supported by the National Natural Science Foundation of China (51174280) and the National Natural Science Foundation of China Youth Fund (51804244).

\section{References}

[1] J. B. Walsh, "The effect of cracks on the compressibility of rock," Journal of Geophysical Research, vol. 70, no. 2, pp. 381-389, 1965.

[2] D. Krajcinovic and M. A. G. Silva, "Statistical aspects of the continuous damage theory," International Journal of Solids and Structures, vol. 18, no. 7, pp. 551-562, 1982.

[3] H. Horii, Nemat, S. Nasser, and M. F. Ashby, "Brittle failure in compression: splitting faulting and brittle-ductile transition," Philosophical Transactions of the Royal Society of London. Series A, Mathematical and Physical Sciences, vol. 319, no. 1549, pp. 337-374, 1986.

[4] Z. C. Tang, "Experimental investigation on temperature-dependent shear behaviors of granite discontinuity," Rock Mechanics and Rock Engineering, vol. 53, no. 9, pp. 40434060, 2020.

[5] Z. C. Tang and Y. Zhang, "Temperature-dependent peak shear-strength criterion for granite fractures," Engineering Geology, vol. 269, Article ID 105552, 2020

[6] G. Li, F. Ma, G. Liu, H. Zhao, and J. Guo, "A strain-softening constitutive model of heterogeneous rock mass considering statistical damage and its application in numerical modeling of deep roadways," Sustainability, vol. 11, no. 8, p. 2399, 2019.

[7] H. Liu and L. Zhang, "A damage constitutive model for rock mass with nonpersistently closed joints under uniaxial compression," Arabian Journal for Science and Engineering, vol. 40, no. 11, pp. 3107-3117, 2015.
[8] G. Wu, K. Wang, M. Zhao, Z. Nie, and Z. Huang, "Analysis of damage evolution of sandstone under uniaxial loading and unloading conditions based on resistivity characteristics," Advances in Civil Engineering, vol. 2019, Article ID 286819, 12 pages, 2019.

[9] Z. C. Tang and Q. Z. Zhang, "Elliptical hertz-based general closure model for rock joints," Rock Mechanics and Rock Engineering, 2020.

[10] Z. C. Tang, Q. Z. Zhang, and J. Peng, "Effect of thermal treatment on the basic friction angle of rock joint," Rock Mechanics and Rock Engineering, vol. 53, no. 4, pp. 1973-1990, 2020.

[11] J. F. Yang, L. Li, and H. J. Lian, "Experimental investigation of the effects of water content on the anisotropy of mode I fracture toughness of bedded mudstones," PLoS One, vol. 15, no. 8, Article ID e0237909, 2020.

[12] J. F. Yang, L. Li, and H. J. Lian, "Experimental evaluation of the influences of water on the fracture toughness of mudstones with bedding," Advances in Materials Science and Engineering, vol. 2019, Article ID 5693654, 16 pages, 2019.

[13] K. Bian, J. Liu, W. Zhang, X. Zheng, S. Ni, and Z. Liu, "Mechanical behavior and damage constitutive model of rock subjected to water-weakening effect and uniaxial loading," Rock Mechanics and Rock Engineering, vol. 52, no. 1, pp. 97-106, 2019.

[14] D. Lei, H. Lin, Y. Chen, R. Cao, and Z. Wen, "Effect of cyclic freezing-thawing on the shear mechanical characteristics of nonpersistent joints," Advances in Materials Science and Engineering, vol. 2019, Article ID 9867681, 14 pages, 2019.

[15] J. Zhang, H. Deng, J. Deng, and H. Guo, "Influence of freezethaw cycles on the degradation of sandstone after loading and unloading," Bulletin of Engineering Geology and the Environment, vol. 79, no. 4, pp. 1967-1977, 2020.

[16] Z. L. Wang, H. Shi, and J. G. Wang, "Mechanical behavior and damage constitutive model of granite under coupling of temperature and dynamic loading," Rock Mechanics and Rock Engineering, vol. 51, no. 10, pp. 3045-3059, 2018.

[17] Z. C. Tang, L. Li, X. C. Wang, and J. P. Zou, "Influence of cyclic freezing-thawing treatment on shear behaviors of granite fracture under dried and saturated conditions," Cold Regions Science and Technology, vol. 181, Article ID 103192, 2021.

[18] D. X. Li, E. Y. Wang, X. G. Kong et al., "Damage precursor of construction rocks under uniaxial cyclic loading tests analyzed by acoustic emission," Construction and Building Materials, vol. 206, no. 6, pp. 169-178, 2019.

[19] S.-Q. Yang and H.-W. Jing, "Strength failure and crack coalescence behavior of brittle sandstone samples containing a single fissure under uniaxial compression," International Journal of Fracture, vol. 168, no. 2, pp. 227-250, 2011.

[20] Z. Zhang, R. Zhang, H. Xie, J. Liu, and P. Were, "Differences in the acoustic emission characteristics of rock salt compared with granite and marble during the damage evolution process," Environmental Earth Sciences, vol. 73, no. 11, pp. 6987-6999, 2015.

[21] C. A. Tang and X. H. Xu, "Evolution and propagation of material defects and kaiser effect function," Journal of Seismological Research, vol. 13, no. 2, pp. 203-213, 1990, in Chinese.

[22] Z. Jia, H. Xie, R. Zhang et al., "Acoustic emission characteristics and damage evolution of coal at different depths under triaxial compression," Rock Mechanics and Rock Engineering, vol. 53, no. 5, pp. 2063-2076, 2020. 
[23] Z. Cui and W. Han, "In SituScanning electron microscope (SEM) observations of damage and crack growth of shale," Microscopy and Microanalysis, vol. 24, no. 2, pp. 107-115, 2018.

[24] X. Y. Wu, P. Baud, and T.-F. Wong, "Micromechanics of compressive failure and spatial evolution of anisotropic damage in Darley Dale sandstone," International Journal of Rock Mechanics and Mining Sciences, vol. 37, no. 1, pp. 143-160, 2000.

[25] G. S. Yang, D. Y. Xie, and C. Q. Zhang, "The quantitative analysis of distribution regulation of CT values of rock damage," Chinese Journal of Rock Mechanics and Engineering, vol. 17, no. 3, pp. 279-285, 1998, in Chinese.

[26] G. S. Yang and H. Liu, "Study on the rock damage characteristics based on the technique of CT image processing," Journal of China Coal Society, vol. 32, no. 5, pp. 463-468, 2007, in Chinese.

[27] Q. S. Zhang, G. S. Yang, and J. X. Ren, "New study of damage variable and constitutive equation of rock," Chinese Journal of Rock Mechanics and Engineering, vol. 22, no. 1, pp. 30-34, 2003, in Chinese.

[28] X. J. Li, X. H. Ni, and Z. D. Zhu, "Statistical damage model for marble under uniaxial compression based on SEM testing scheme," Journal of Hydroelectric Engineering, vol. 34, no. 5, pp. 119-123, 2015, in Chinese.

[29] H. Jia, W. Xiang, and M. Krautblatter, "Quantifying rock fatigue and decreasing compressive and tensile strength after repeated freeze-thaw cycles," Permafrost and Periglacial Processes, vol. 26, no. 4, pp. 368-377, 2015.

[30] X. H. Ni, Z. D. Zhu, J. Zhao, D. W. Li, and X. T. Feng, "Mesodamage mechanical digitalization test of complete process of rock failure," Rock and Soil Mechanics, vol. 30, no. 11, pp. 3283-3290, 2009, in Chinese.

[31] Y. Y. Cao, S. P. Ma, X. Wang, and Z. Y. Hong, "A new definition of damage variable for rock material based on the spatial characteristics of deformation fields," Advanced Materials Research, vol. 146, no. 4, pp. 865-868, 2010.

[32] S. P. Ma, S. J. Liu, and Y. H. Zhao, "Gray correlation of digital images from loaded rock specimen surface to evaluate its damage evolution," Chinese Journal of Rock Mechanics and Engineering, vol. 25, no. 3, pp. 590-595, 2006, in Chinese.

[33] X. B. Wang, W. T. Hou, Y. S. Pan, and W. Dong, "Experiments of strain localization processes of coal specimens in uniaxial compression based on the digital image correlation method," Journal of China Coal Society, vol. 43, no. 04, pp. 984-992, 2018, in Chinese.

[34] G. Yang, Z. Cai, X. Zhang, and D. Fu, "An experimental investigation on the damage of granite under uniaxial tension by using a digital image correlation method," Optics and Lasers in Engineering, vol. 73, no. 4, pp. 46-52, 2015.

[35] F. P. Zhu, D. Y. Kong, Y. Gong et al., "The measurement of tensile mechanical properties of high-strength steel using three-dimensional digital image correlation," Chinese Quarterly of Mechanics, vol. 39, no. 2, 2018, in Chinese.

[36] GBT 50266-2013, Standard for Test Methods of Engineering Rock Mass, China Planning Press, Beijing, China, 2013, in Chinese.

[37] Q. Li, M. Chen, Y. Jin, and B. Zhang, "Indoor evaluation method for shale brittleness and improvement," Chinese Journal of Rock Mechanics and Engineering, vol. 31, no. 8, pp. 1681-1685, 2012, in Chinese.

[38] H. Wang, S. Yu, H. Li, R. Ren, L. Tang, and W. Zhu, "Ultrasonic fracturing of brittle solids with an internal crack based on 3d-ILC method," Chinese Journal of Rock Mechanics and Engineering, vol. 39, no. 5, pp. 938-948, 2020, in Chinese. 